\title{
Theoretical and experimental investigation on the intersystem crossing kinetics in benzothioxanthene imide luminophores, and their dependence on substituents effects.
}

Laura Abad Galán, ${ }^{\mathrm{a}}$ José M. Andrés Castán, ${ }^{\mathrm{b}}$ Clément Dalinot, ${ }^{\mathrm{b}}$ Pablo Simón Marqués, ${ }^{\mathrm{b}}$ Philippe Blanchard, ${ }^{\mathrm{b}}$ Olivier Maury, ${ }^{\mathrm{a}}$ Clément Cabanetos, ${ }^{\mathrm{b} *}$ Tangui Le Bahers, ${ }^{\mathrm{a} *}$ Cyrille Monnereau ${ }^{\mathrm{a} *}$

${ }^{a}$ Univ Lyon, ENS de Lyon, CNRS UMR 5182, Université Claude Bernard Lyon 1, F-69342 Lyon, France.

${ }^{\mathrm{b}}$ CNRS UMR 6200, Moltech-Anjou, University of Angers, 2 Bd Lavoisier, 49045 Angers, France

\begin{abstract}
In spite of their remarkable luminescence properties, benzothioxanthene imide (BTXI an imide containing rylene chromophores) derivatives have been largely overlooked compared to their perylene bisimide and naphthalene bisimide counterparts. Thus their detailed photophysics are much less understood. In this paper, we show how relatively simple structural modifications of the backbone of BTXIs can lead to impressive variations in their Inter-System Crossing kinetics. Thus, through rational engineering of their structure, it is possible to obtain a triplet formation quantum yield that reaches unity, making BTXI a promising class of compounds for triplet-based applications (photodynamic therapy, electroluminescence, etc.).
\end{abstract}

\section{Introduction}

Rylene imide derivatives are a very intriguing subclass of extended $\pi$-conjugated dyes which have been thoroughly studied for various organic electronic applications, ${ }^{1,2}$ such as electroluminescent devices, ${ }^{3,}$ ${ }^{4}$ field effect transistors, ${ }^{5,6}$ photovoltaics ${ }^{7-10}$ or luminescent tags for bioimaging. ${ }^{11-13}$ Their success mostly results from the interplay of outstanding spectroscopic properties such as high luminescence quantum yields, ${ }^{14}$ a large range of structure dependent accessible emission wavelengths, ${ }^{15}$ high thermal and photostability ${ }^{16}$ and associated to a moderate synthetic cost compatible with large scale syntheses. ${ }^{17,18}$ Within this rylene family, the benzothioxanthene imide derivatives (BTXI) constitute a largely overlooked class of chromophore, ${ }^{19-23}$ especially when compared to perylene bisimide. Recently, we have demonstrated that because of their relative synthetic ease, and the possibility to introduce a variety of functional groups on the $\beta$-position of the naphthyl unit with regards to the sulphur atom (position 5 in Figure 1)position of the naphthalimide moieties, BTXI derivatives could be used as relevant building blocks to prepare extended p-functional molecular systems. ${ }^{24,25}$

As for other rylenes, the photophysical properties of BTXI derivatives are clearly remarkable. As a matter of fact, in spite of its apparent structural simplicity, the BTXI core in its most simple form (BTXI, Figure 1) combines large extinction coefficient in the blue part of the electromagnetic spectrum, along with near unity emission quantum yields in the yellow-green domain. ${ }^{26,27}$ Moreover, in the course of these preliminary studies on the spectroscopic properties of BTXI derivatives, we identified that the excited state photophysics of this class of chromophore were extremely sensitive to their substitution 
pattern. ${ }^{28}$ In particular, singlet-to-triplet Inter-System Crossing (ISC) seemed to be consistently increased upon simple substitution with a variety of groups. Quite remarkably, this evolution was not limited to halogen substituents, as often observed in organic molecules owing to the so-called heavy atom effects. Strong substituents effects were also observed with generally more innocent functional groups such as nitro, amino, or diphenylamino. A similar observation has also been reported by Zhang et al. on a particular extended BTXI derivative. ${ }^{29}, 30$ Besides, we demonstrated that simple asfunctionalised molecules could efficiently sensitise the production of singlet oxygen, with potential applications in photocatalysis or photodynamic therapy (PDT). ${ }^{31,32}$

Triggered by this initial observation, we initiated a more detailed investigation of the mechanisms responsible for such dramatic dependence of ISC kinetics on the substitution parameters. We were particularly eager to study whether we could amplify this effect by appropriate modification of the substitution pattern, beyond simple tuning of the position 5 . In order to do so, we initiated a coupled theoretical and experimental study to elucidate the molecular features that impact the spin-orbit coupling (SOC) in this class of compounds. Thus, in addition to the molecules previously reported, where only modification of the position 5 was considered, we investigated other possible modifications: complementary substitution on the bay position (position 11) were investigated; we also looked at the effect of oxidation of the sulfur heteroatom of the thioxanthene moieties, leading to the thioxanthene dioxide (Figure 1). For all synthesised compounds, we monitored in detail the effect of substitution on the spectroscopic and photophysical features of the chromophore (energy of the absorption and emission, fluorescence quantum yield, singlet oxygen generation quantum yield). Additionally, most of the studied molecules were investigated by computational modeling, mostly aiming at rationalizing the kinetics of ISC on the basis of the calculated spin-orbit coupling matrix and difference in energy between the first singlet excited states and triplet excited states. As a result, three main conclusions can be drawn from this study: (i) chemical modifications on the BTXI core are extremely efficient to tune the extent of ISC within the molecule, allowing to reach a quantum yield in triplet formation that approaches unity; (ii) the influence of the substitution on the ISC kinetics does not exclusively relies on classical heavy atom effects, but to a more complex interplay, involving molecular distortion and (iii) this last effect, and its influence on the ISC kinetics, depends strongly on the nature of the substituents. 


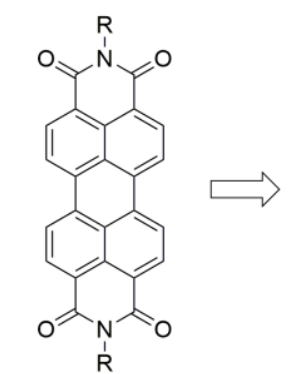

Perylene bisimide

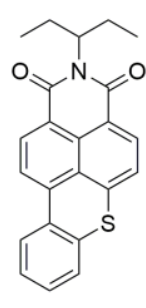

BTXI

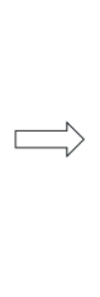

(11)

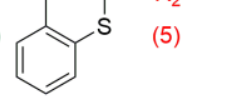

BTXI-R ${ }_{1} \mathbf{R}_{2}$

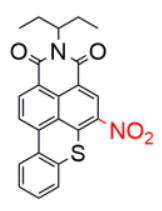

BTXI-NO

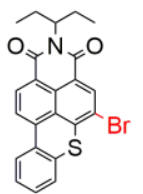

BTXI-Br

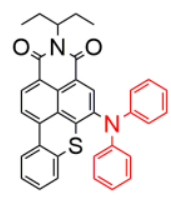

BTXI-N $(\mathrm{Ph})_{2}$

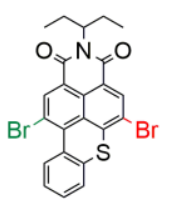

BTXI-BrBr

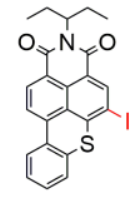

BTXI-I

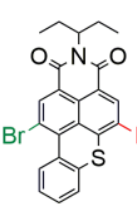

BTXI-BrI<smiles>C=C=CC(CC)N1C(=O)c2ccc3c4c(ccc(c24)C1=O)S(=O)(=O)c1ccccc1-3</smiles>

BTXI-SO $_{2}$

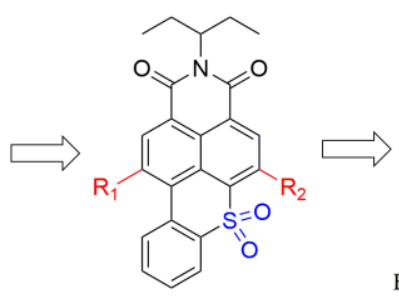

BTXI-SO ${ }_{2}-\mathrm{R}_{1} \mathbf{R}_{2}$

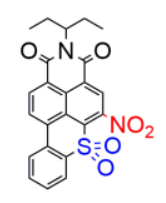

BTXI- $-\mathrm{SO}_{2}-\mathrm{NO}_{2}$
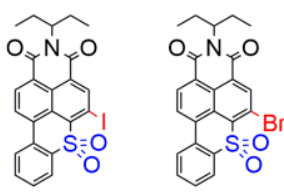

BTXI-SO 2 -Br

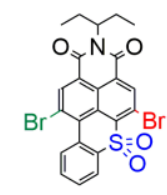

BTXI-SO ${ }_{2}-\mathrm{BrBr}$

Figure 1. Representation of the well-studied perylenebisimide molecule, the BTXI core and the derivatives studied in this report.

\section{Experimental}

\section{Synthesis}

All reagents and chemicals from commercial sources were used without further purification. Solvents were dried and purified using standard techniques. Flash chromatography was performed with analytical-grade solvents using Aldrich silica gel (technical grade, pore size $60 \AA$, 230-400 mesh particle size). Flexible plates ALUGRAM $^{\circledR}$ Xtra SIL G UV254 from MACHEREY-NAGEL were used for TLC. Compounds were detected by UV irradiation (Bioblock Scientific). NMR spectra were recorded with a Bruker AVANCE III 300 (1H, $300 \mathrm{MHz}$ and 13C, 75MHz) or a Bruker AVANCE DRX500 (1H, $500 \mathrm{MHz}$; 13C, $125 \mathrm{MHz}$ ). Chemical shifts are given in ppm relative to TMS and coupling constants $\mathrm{J}$ in $\mathrm{Hz}$. IR spectra were recorded on a Bruker spectrometer Vertex 70 and UV-vis spectra with a Perkin Elmer 950 spectrometer. Matrix Assisted Laser Desorption/Ionization was performed on MALDI-TOF MS BIFLEX III Bruker Daltonics spectrometer using dithranol as matrix. High resolution mass spectrometry (HRMS) was performed with a JEOL JMS-700 B/E.

Synthetic procedures to prepare BTXI, BTXI-Br, BTXI-BrBr, BTXI-I, BTXI-BrI, BTXI-NO ${ }_{2}$ and $\mathbf{B T X I - N P \mathbf { N } _ { 2 }}$ are accessible from our early reported publications. ${ }^{24,28,33}$

General procedure for the synthesis of $\mathrm{BTXI}-\mathrm{SO}_{2}$ derivatives: a solution of $m$-Chloroperbenzoic acid ( $m$ CPBA) (4 eq) in $\mathrm{CH}_{2} \mathrm{Cl}_{2}$ was added dropwise on a stirred solution of BTXI (1 eq) in $\mathrm{CH}_{2} \mathrm{Cl}_{2}$. After a night of stirring at room temperature, the reaction was quenched with a saturated solution of $\mathrm{Na}_{2} \mathrm{~S}_{2} \mathrm{O}_{3}$. The organic phase was then subsequently extracted with dichloromethane, washed with a saturated solution of $\mathrm{NaHCO}_{3}$ before being dried with $\mathrm{MgSO}_{4}$ and finally concentrated under vacuum. 
BTXI-SO $\mathrm{S}_{2}$ : The crude was purified by column chromatography on silica gel (eluent: $\mathrm{CH}_{2} \mathrm{Cl}_{2}$ ) affording a pale yellow solid in quantitative yield (100\% yield). ${ }^{1} \mathrm{H}$ NMR $\left(300 \mathrm{MHz}, \mathrm{CDCl}_{3}\right): \delta(\mathrm{ppm}) 8.86-8.76(\mathrm{~m}$, $2 \mathrm{H}), 8.68-8.62(\mathrm{~m}, 2 \mathrm{H}), 8.37-8.30(\mathrm{~m}, 2 \mathrm{H}), 7.83(\mathrm{td}, \mathrm{J}=7.7,1.6 \mathrm{~Hz}, 1 \mathrm{H}), 7.75(\mathrm{td}, \mathrm{J}=7.6,1.2 \mathrm{~Hz}, 1 \mathrm{H})$, $5.11-4.94(\mathrm{~m}, 1 \mathrm{H}), 2.34-2.13(\mathrm{~m}, 2 \mathrm{H}), 2.03-1.84(\mathrm{~m}, 2 \mathrm{H}), 0.91(\mathrm{t}, \mathrm{J}=7.5 \mathrm{~Hz}, 6 \mathrm{H}) .{ }^{13} \mathrm{C} \mathrm{NMR}(75 \mathrm{MHz}$, $\left.\mathrm{CDCl}_{3}\right): \delta$ (ppm) 138.1, 136.3, 133.6, 132.2, 131.1, 130.8, 130.3, 128.6, 126.5, 125.3, 124.9, 124.8, 123.4, 58.3, 25.1, 11.4. HRMS (EI): $\mathrm{m} / \mathrm{z}$ calcd for $\mathrm{C}_{23} \mathrm{H}_{19} \mathrm{NO}_{4} \mathrm{~S}: 405.1029$, found: 405.1031 .

BTXI-SO $-\mathrm{Br}$ : The crude was purified by column chromatography on silica gel (eluent: $\mathrm{CH}_{2} \mathrm{Cl}_{2}$ ) affording a pale yellow solid in $74 \%$ yield. ${ }^{1} \mathrm{H} \mathrm{NMR}\left(300 \mathrm{MHz}, \mathrm{CDCl}_{3}\right): \delta(\mathrm{ppm}) 8.91(\mathrm{~s}, 1 \mathrm{H}), 8.74(\mathrm{~d}, \mathrm{~J}=8.0 \mathrm{~Hz}, 1 \mathrm{H})$, $8.64(\mathrm{~d}, \mathrm{~J}=8.0 \mathrm{~Hz}, 1 \mathrm{H}), 8.33-8.26(\mathrm{~m}, 2 \mathrm{H}), 7.84-7.72(\mathrm{~m}, 2 \mathrm{H}), 5.08-4.93(\mathrm{~m}, 1 \mathrm{H}), 2.30-2.13(\mathrm{~m}$, 2H), $2.01-1.84(\mathrm{~m}, 2 \mathrm{H}), 0.90$ (t, J = 7.5 Hz, 6H). ${ }^{13} \mathrm{C} \mathrm{NMR}\left(75 \mathrm{MHz}, \mathrm{CDCl}_{3}\right): \delta$ (ppm) 137.6, 136.6, 136.2, 133.6, 131.9, 131.3, 129.6, 129.5, 127.7, 126.0, 125.6, 125.6, 125.3, 121.7, 58.5, 25.0, 11.4. HRMS (EI): $\mathrm{m} / \mathrm{z}$ calcd for $\mathrm{C}_{23} \mathrm{H}_{18} \mathrm{BrNO}_{4} \mathrm{~S}: 483.0134$, found: 483.0134 .

BTXI-SO $-\mathrm{NO}_{2}$ : The crude was purified by column chromatography on silica gel (eluent: $\mathrm{CH}_{2} \mathrm{Cl}_{2}$ ) affording a pale yellow solid in $74 \%$ yield. ${ }^{1} \mathrm{H}$ NMR $\left(300 \mathrm{MHz}, \mathrm{CDCl}_{3}\right): \delta(\mathrm{ppm}) 8.87(\mathrm{~d}, \mathrm{~J}=8.0 \mathrm{~Hz}, 1 \mathrm{H})$, $8.78(\mathrm{~s}, 1 \mathrm{H}), 8.73(\mathrm{~d}, \mathrm{~J}=8.0 \mathrm{~Hz}, 1 \mathrm{H}), 8.31(\mathrm{dd}, \mathrm{J}=7.8,1.4 \mathrm{~Hz}, 1 \mathrm{H}), 8.24(\mathrm{dd}, \mathrm{J}=7.8,1.0 \mathrm{~Hz}, 1 \mathrm{H}), 7.85(\mathrm{td}$, $\mathrm{J}=7.8,1.4 \mathrm{~Hz}, 1 \mathrm{H}), 7.77(\mathrm{td}, \mathrm{J}=7.8,1.0 \mathrm{~Hz}, 1 \mathrm{H}), 5.10-4.90(\mathrm{~m}, 1 \mathrm{H}), 2.32-2.13(\mathrm{~m}, 2 \mathrm{H}), 2.01-1.81$ $(\mathrm{m}, 2 \mathrm{H}), 0.90(\mathrm{t}, \mathrm{J}=7.5 \mathrm{~Hz}, 6 \mathrm{H}) .{ }^{13} \mathrm{C} \mathrm{NMR}\left(75 \mathrm{MHz}, \mathrm{CDCl}_{3}\right): \delta(\mathrm{ppm}) 146.7,136.1,134.2,134.0,132.1$, 131.3, 130.5, 129.1, 129.0, 127.1, 126.9, 125.1, 125.0, 124.5, 58.8, 25.0, 11.4. HRMS (EI): $\mathrm{m} / \mathrm{z}$ calcd for $\mathrm{C}_{23} \mathrm{H}_{18} \mathrm{~N}_{2} \mathrm{O}_{6} \mathrm{~S}: 450.0880$, found: 450.0886 .

BTXI-SO - -I: The crude was purified by column chromatography on silica gel (eluent: $\mathrm{CH}_{2} \mathrm{Cl}_{2}$ ) affording a pale yellow solid in $31 \%$ yield. ${ }^{1} \mathrm{H} N M R\left(500 \mathrm{MHz}, \mathrm{CDCl}_{3}\right): \delta(\mathrm{ppm}) 9.23(\mathrm{~s}, 1 \mathrm{H}), 8.75(\mathrm{~d}, \mathrm{~J}=8.0 \mathrm{~Hz}, 1 \mathrm{H})$, $8.61(\mathrm{~d}, \mathrm{~J}=8.2 \mathrm{~Hz}, 1 \mathrm{H}), \delta 8.32(\mathrm{dd}, \mathrm{J}=7.8,1.5 \mathrm{~Hz}, 1 \mathrm{H}), 8.27(\mathrm{~d}, \mathrm{~J}=7.8,1 \mathrm{H}), 7.80(\mathrm{td}, \mathrm{J}=7.6,1.2 \mathrm{~Hz}, 1 \mathrm{H})$, $7.75(\mathrm{td}, \mathrm{J}=7.6,1.2 \mathrm{~Hz}, 1 \mathrm{H}), 5.00(\mathrm{~m}, 1 \mathrm{H}), 2.28-2.15(\mathrm{~m}, 2 \mathrm{H}), 1.98-1.86(\mathrm{~m}, 2 \mathrm{H}), 0.90(\mathrm{t}, \mathrm{J}=7.5 \mathrm{~Hz}$, $6 \mathrm{H}) .{ }^{13} \mathrm{C} \mathrm{NMR}\left(125 \mathrm{MHz}, \mathrm{CDCl}_{3}\right): \delta$ (ppm) 144.6, 139.2, 135.9, 133.7, 132.1, 131.2, 129.8, 129.1, 128.3, $125.8,125.5,125.3,91.0,58.5,25.1,11.4$. HRMS (FAB): calcd for $\mathrm{C}_{23} \mathrm{H}_{18} \mathrm{INO}_{4} \mathrm{~S}$ : 531.0001, found: 531.0002 .

BTXI-SO ${ }_{2}-\mathrm{BrBr}$ : The crude was purified by column chromatography on silica gel (eluent: $\mathrm{CH}_{2} \mathrm{Cl}_{2}$ ) affording a pale yellow solid in $19 \%$ yield. ${ }^{1} \mathrm{H}$ NMR $\left(300 \mathrm{MHz}, \mathrm{CDCl}_{3}\right): \delta(\mathrm{ppm}) 8.93(\mathrm{~s}, 1 \mathrm{H}), 8.79(\mathrm{~s}, 1 \mathrm{H})$, $8.71-8.62(\mathrm{~m}, 1 \mathrm{H}), 8.43-8.31(\mathrm{~m}, 1 \mathrm{H}), 7.80-7.69(\mathrm{~m}, 2 \mathrm{H}), 4.97(\mathrm{~m}, 1 \mathrm{H}), 2.27-2.11(\mathrm{~m}, 2 \mathrm{H}), 1.99-$ $1.85(\mathrm{~m}, 2 \mathrm{H}), 0.89(\mathrm{t}, \mathrm{J}=7.5 \mathrm{~Hz}, 6 \mathrm{H}) .{ }^{13} \mathrm{C} \mathrm{NMR}\left(75 \mathrm{MHz}, \mathrm{CDCl}_{3}\right): \delta(\mathrm{ppm}) 138.4,137.6,135.8,135.3$, $132.7,132.1,131.3,130.9,130.7,129.0,126.2,125.9,123.5,121.7,58.7,25.0,11.3$. HRMS (FAB): calcd for $\mathrm{C}_{23} \mathrm{H}_{17} \mathrm{Br}_{2} \mathrm{NO}_{4} \mathrm{~S}$ : 560.9245 , found: 560.9238 .

\section{Photophysical measurements}

Absorption spectra were recorded on a JASCO V-650 spectrophotometer in diluted solution (ca. $10^{-5}$ or $10^{-6} \mathrm{~mol} \mathrm{~L}^{-1}$ ), using spectrophotometric grade solvents. Emission spectra were measured using Horiba-Jobin-Yvon Fluorolog-3 fluorimeter. The steady-state luminescence was excited by unpolarised light from a $450 \mathrm{~W}$ xenon continuous wave (CW) lamp and detected at an angle of $90^{\circ}$ for measurements of dilute solutions ( $10 \mathrm{~mm}$ quartz cuvette) by using a Hamamatsu R928. Spectra were corrected for both excitation source light-intensity variation and emission spectral responses. Luminescence quantum yields $Q$ were measured in diluted solutions with an absorbance lower than 0.1 , by using the following Equation 1 : 


$$
\frac{Q x}{Q r}=\left[\frac{A r(\lambda)}{A x(\lambda)}\right] \cdot\left[\frac{n_{x}^{2}}{n_{r}^{2}}\right] \cdot\left[\frac{D x}{D r}\right]
$$

where $A(\lambda)$ is the absorbance (or optical density) at the excitation wavelength, $n$ the refractive index of the solvent and $D$ the integrated luminescence intensity. " $r$ " and " $x$ " stand for reference and sample, respectively. Here, the reference is coumarin-153 in methanol $(Q r=0.45) \cdot{ }^{34}$ Excitations of reference and sample compounds were performed at the same wavelength. The reported results are the average of 4-5 independent measurements at various absorbances (comprised between 0.01-0.1) for both sample and reference. The plot of the integrated luminescence intensity vs. absorbance gives straight line with excellent correlation coefficients and the slope $S$ can be determined for both sample $(x)$ and reference ( $r$ ). Equation 1 becomes Equation 2.

$$
\frac{Q x}{Q r}=\left[\frac{S x}{S r}\right] \cdot\left[\frac{n_{x}^{2}}{n_{r}^{2}}\right]
$$

For singlet oxygen quantum yield determination $\phi_{\Delta}$, the principle is exactly the same except that the singlet oxygen luminescence emission band (D) is integrated for both sample $(x)$ and reference $(r)$ compounds. $A(\lambda)$ is the absorbance (or optical density) at the excitation wavelength. In this case it is very important that both experiments are conducted in the same solvent at exactly the same excitation wavelength $(n x=n r)$. The reported results are the average of 4-5 independent measurements at various absorbances (comprised between 0.01-0.1) for both sample and reference. The plot of the integrated singlet oxygen luminescence intensity vs. absorbance gives straight line with excellent correlation coefficients and the slope $\mathrm{S}$ can be determined for both sample $(\mathrm{x})$ and reference $(r)$. In the present case, the reference is phenalenone $\left(\phi_{\Delta r}=0.98\right.$ in dichloromethane). ${ }^{35}$

Excited state lifetime analyses were measured at the maximum emission wavelength using a 390nm nanoLED by means of the time correlated single photon counting (TCSPC) method of diluted dichloromethane solutions at room temperature.

\section{Computational details}

Molecular calculations were carried out with the Gaussian 16 code. ${ }^{36}$ The global hybrid functional PBEO was used both for ground state and excited state geometry optimisations ${ }^{37}$ This functional was chosen because of its frequently reported accuracy for modelling the localised electronic transitions in organic molecules. ${ }^{38}$ Structural optimisations and subsequent frequency calculations for both the ground and excited states were performed using an all electron Pople triple zeta basis set with one polarisation function on all atoms and one diffuse function of heavier atoms, known as $6-311+G(d, p)$, for $H, C, N$, $\mathrm{O}, \mathrm{S}$ and $\mathrm{Br}$ atoms. ${ }^{39}$ For I atoms, the aug-cc-pVDZ basis set along with the associated pseudo-potential was used. Bulk solvent effects were included using the Polarizable Continuum Model (PCM) of Tomasi and co-workers. ${ }^{40}$ Default radii (from the UFF, scaled by 1.1) were used. Transitions in absorption were simulated by computing the first 10 transitions in TD-DFT at the same level of theory. The fluorescence energies were obtained by relaxing the first excited state geometry in TD-DFT at the same level of theory.

The Dalton ${ }^{41}$ program was used to compute the SOC between the two first triplet states (namely $T_{1}$ and $T_{2}$ ) and the $S_{1}$ state at the $S_{1}$ optimised geometry using the quadric-response TD-DFT at the B3LYP/PCM level with the aug-cc-pVDZ basis set adapted for the Douglas-Kroll calculations. ${ }^{42}$ The SpinOrbit Coupling was computed using the Douglas-Kroll Hamiltonian along with the spin-orbit mean field approach. ${ }^{43}$ 
Vibrationally resolved spectra were obtained, after performing frequency calculations for the ground and excited states, using the FC classes program. ${ }^{44}$ The reported spectra were simulated at $0 \mathrm{~K}$ using convolution Gaussian function presenting a FWHM of 0.05 eV. 25 overtones for each mode and 20 combination bands were used as a maximum of $10^{10}$ integrals for each class.

\section{Results and discussion}

Synthesis

BTXI, BTXI-Br, BTXI-BrBr, BTXI-I, BTXI-BrI, BTXI-NO ${ }_{2}$ and BTXI-NPh ${ }_{2}$ were prepared according to our

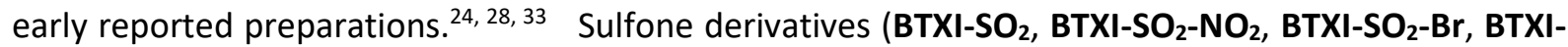

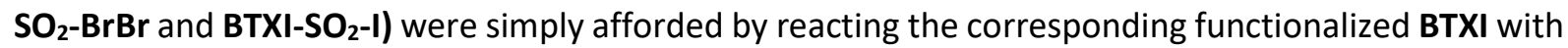
$m$-Chloroperbenzoic acid ( $m C P B A$ ) in dichloromethane, overnight and at room temperature. ${ }^{20}$

\section{Spectroscopy}

All molecules were studied in diluted dichloromethane solutions and systematically evaluated regarding their absorption, emission wavelength, emission quantum yield and lifetime. The generation of singlet oxygen, as a key parameter of this study, was measured by directly looking at the phosphorescence band of singlet oxygen centered at $1270 \mathrm{~nm}$. Finally, the triplet state of all molecules was also assessed when possible, by checking the phosphorescence spectrum in glass medium (ethanol/methanol 4:1 at 77K) after applying a $0.05 \mathrm{~ms}$ delay to filter the short component of emission coming from fluorescent states. (Table 1 and Figures 2,3,4 and S1-S21).

Table 1. Summary of the main spectroscopic and photophysical data for all studied BTXI derivatives.

\begin{tabular}{|c|c|c|c|c|c|c|}
\hline & $\lambda_{\mathrm{abs}(\max )}(\mathrm{nm})$ & $\lambda_{\text {em }}(\mathrm{nm})$ & $\Phi_{f}^{a}$ & $\tau_{\text {obs }}(\mathrm{ns})$ & $\Phi_{\Delta}^{b}$ & $E_{\text {Triplet }}\left(\mathrm{cm}^{-1}\right)^{c}$ \\
\hline BTXI & 455 & 510 & 0.99 & 7.48 & - & - \\
\hline BTXI-Br & 469 & 500 & 0.78 & 6.49 & 0.11 & 14925 \\
\hline BTXI-BrBr & 468 & 530 & 0.60 & 7.15 & 0.23 & 14450 \\
\hline BTXI-I & 471 & 505 & 0.58 & 4.74 & 0.37 & 14950 \\
\hline BTXI-BrI & 471 & 535 & 0.39 & 4.68 & 0.70 & 14350 \\
\hline BTXI-NO & 486 & 585 & 0.01 & 8.14 & 0.42 & 15870 \\
\hline BTXI-NPh 2 & 483 & 600 & 0.33 & 10.68 & 0.46 & $14600-$ \\
\hline BTXI-SO & 379 & 442 & 0.15 & $<1^{d}$ & 0.72 & 16250 \\
\hline BTXI-SO ${ }_{2}-\mathrm{NO}_{2}$ & 396 & 495 & 0.01 & $<1^{d}$ & 0.57 & - \\
\hline $\mathrm{BTXI}-\mathrm{SO}_{2}-\mathrm{Br}$ & 386 & 455 & 0.06 & $<1^{d}$ & 0.97 & 15550 \\
\hline BTXI-SO ${ }_{2}-\mathrm{BrBr}$ & 388 & 474 & 0.02 & $<1^{d}$ & 1.00 & 15200 \\
\hline BTXI-SO ${ }_{2}-\mathrm{I}$ & 380 & 446 & 0.01 & $<1^{d}$ & 1.00 & 15500 \\
\hline
\end{tabular}

${ }^{a}$ Measured using Coumarin-153 as reference $\left(\Phi_{\mathrm{F}}=0.45\right.$ in methanol).

${ }^{\mathrm{b}}$ Measured using Phenalenone as reference $\left(\Phi_{\mathrm{F}}=0.95\right.$ in dichloromethane).

${ }^{\mathrm{c}}$ Calculated at the zero-phonon transition correspondently marked with *. 


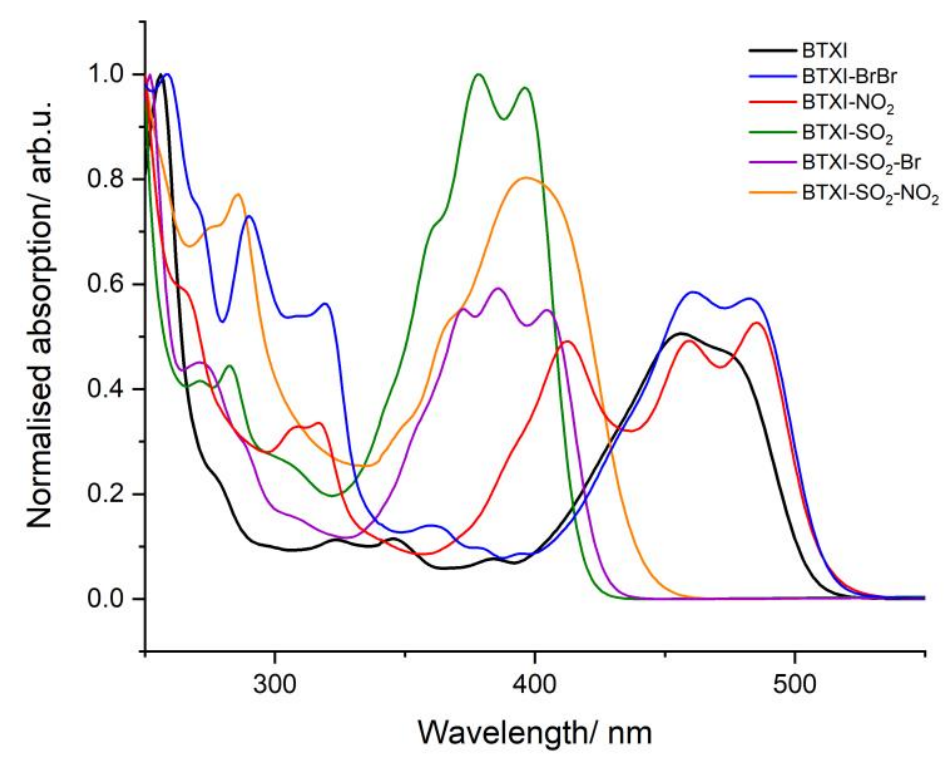

Figure 2. Normalised absorption spectra of the selected BTXI derivatives in diluted dichloromethane solutions at room temperature.

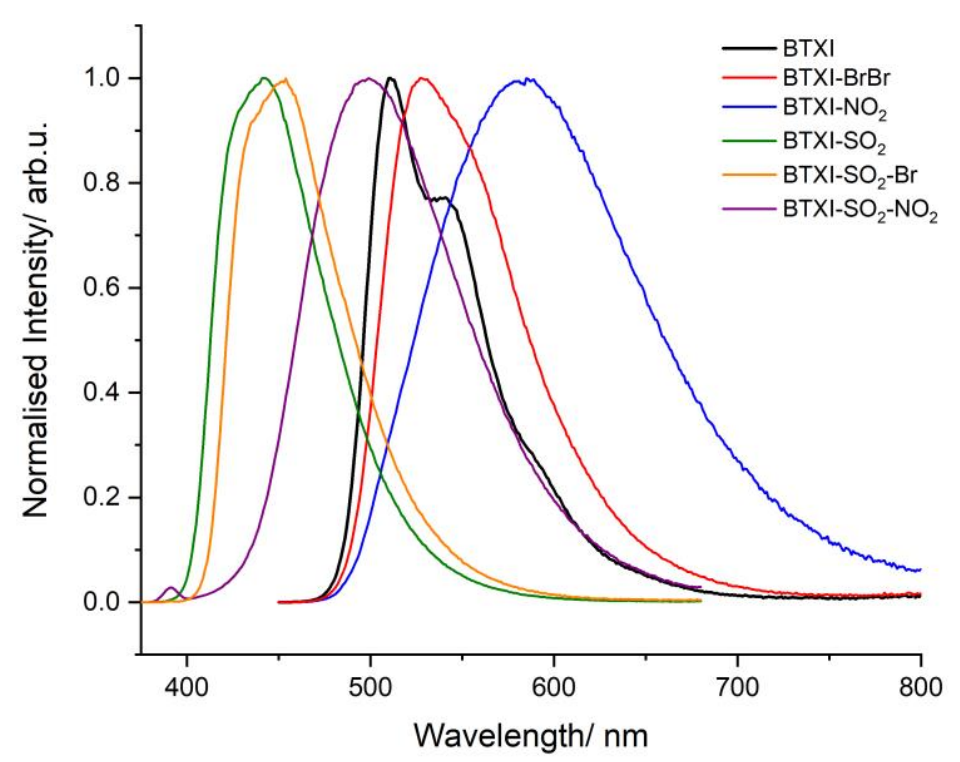

Figure 3. Normalised emission spectra of the selected BTXI derivatives in diluted dichloromethane solutions at room temperature.

All compounds present similarities in their spectral signature: absorption is dominated by a broad yet structured intense band, whose position is slightly affected by the presence and nature of the 
substituents in position 5 , but undergoes drastic evolution upon oxidation of the sulfur atom (vide infra). Emission is characterised by a moderate Stokes shift, which tends to increase upon nitro substitution in both the sulfur and sulfone series. Along with the broadening and complete loss of vibronic structure of the band, this suggests a transition in the character of the emissive state from a

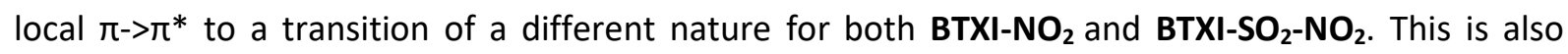

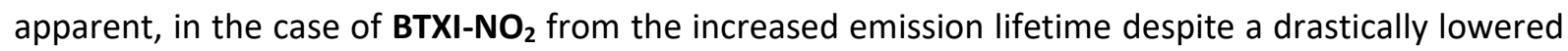
emission quantum yield, typical of a forbidden nature of the associated transition. Based on TD-DFT calculations, we can confirm the $\pi->\pi^{*}$ and local character of the first $S_{0} \rightarrow S_{1}$ transition for almost all the molecules investigated here, characterised by $\mathrm{D}_{\mathrm{CT}}$ around $2.5 \AA$ for the BTXI series and around 3.1

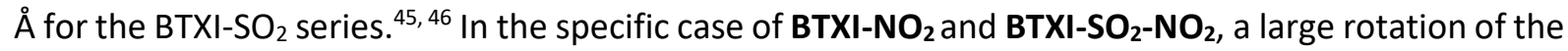
$-\mathrm{NO}_{2}$ group at the $\mathrm{S}_{1}$ state first makes the transition $\mathrm{S}_{0} \rightarrow \mathrm{S}_{1}$ out of the Franck-Condon region resulting in the loss of the band's structure and then changes the nature of the transition from a $\pi->\pi^{*}$ to a $n-$ $>\pi^{*}$ with a computed 0.0 oscillator strength explaining the long life time and the low fluorescence efficiency for the nitrated molecules. For interested readers, a comparison between simulated and experimental spectra is given in Supporting Information. Although quantitative in the native BTXI compound, luminescence quantum yield drops with substitution. In the sulfur series, except for BTXI$-\mathrm{NO}_{2}$, a good reverse correlation exists between the decrease in luminescence quantum yield and the increase in singlet oxygen generation (vide infra). On the other hand, in the sulfone series, luminescence is fully quenched for all compounds as shown by the low values of quantum yields and the short excited state lifetimes.

Already reported in one of our previous paper, the brominated $\mathbf{B T X I - B r}$ (singlet oxygen generation

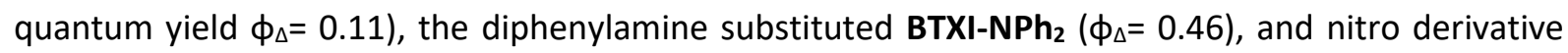
BTXI-NO ${ }_{2}\left(\phi_{\Delta}=0.44\right)$ were shown to be efficient singlet oxygen sensitizers due to important spin orbit coupling (SOC) (as also manifested form the observation of a distinctive phosphorescence signal at $77 \mathrm{~K}$ ) as it will be confirmed by quantum chemistry later in this article. Herein, we extend our investigation on the effect of halogen substitution on the ISC parameters. First, the bromine atome of the BTXI-Br in was replaced by an iodine atom. It turns out that while this substitution had only marginal effect on the position and shape of the absorption and emission band in the resulting BTXI-I molecule, a significant decrease of the emission quantum yield (from 0.78 to 0.58 ) and lifetime (from 6.5 to $4.7 \mathrm{~ns}$ ) was observed, with a concomitant increase in singlet oxygen generation efficiency (from 0.11 to 0.37 ), thus witnessing a more pronounced effect of the heavier iodine atom as compared to bromine. Introduction of a second halogen atom in the bay position was thereafter investigated, revealing a marked increase in the ISC kinetics along with the heavy atom effect. Compared to BTXI$\mathrm{Br}$, addition of another bromine substituent ( $\mathrm{BTXI-BrBr} \phi_{\Delta}=0.23$ ) was indeed less effective in boosting singlet oxygen generation efficiency than substituting the bay position with an iodine (BTXI-Brl $\phi_{\Delta}=$ $0.70)$. 
a)

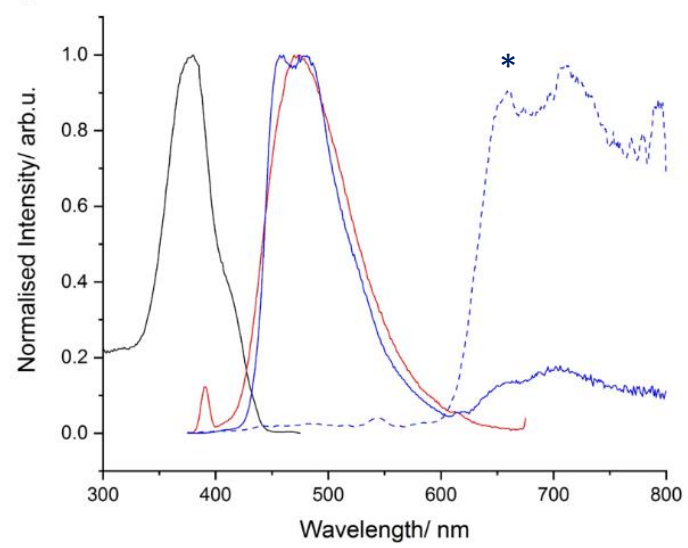

b)

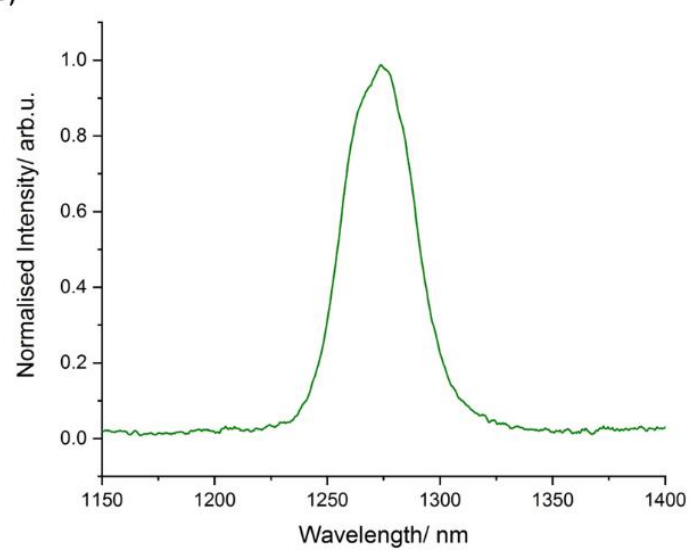

Figure 4. a) Normalised excitation ( $\lambda_{\mathrm{em}}=500 \mathrm{~nm}$ ) (black trace), emission $\left(\lambda_{\mathrm{exc}}=375 \mathrm{~nm}\right.$ ) at room temperature (red trace) and $77 \mathrm{~K}$ (blue trace) without (full trace) or with a delay of $0.05 \mathrm{~ms}$ delay

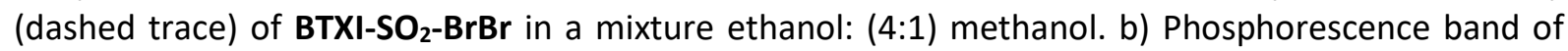
singlet oxygen observed for $\mathbf{B T X I}-\mathrm{SO}_{2}-\mathrm{BrBr}$ in a diluted dichloromethane solution at room temperature.

Then, we systematically compared the sulfone-modified analogues of BTXI to their parent molecules. Quite remarkably, oxidation of the sulfur bridge did not only result in a marked change in the position of the absorption and emission maxima ( $c a .80-90 \mathrm{~nm}$ blue shift of both bands upon oxidation, depending on the nature of the substituents with respect to their reference molecule) but also to a tremendous effect on their ISC efficiencies. While the BTXI features a luminescence quantum yield near unity and does not promote any measurable singlet oxygen generation, its oxidized version, namely the $\mathbf{B T X I - S O _ { 2 }}$ experiences a drastic drop in luminescence efficiency $\left(\phi_{\mathrm{em}}=0.15\right)$ that goes with a burst in singlet oxygen efficiency, reaching an impressive value of 0.72. Even more remarkably, cumulative effect is achieved upon additional substitution of the BTXI core with halogen substituents,

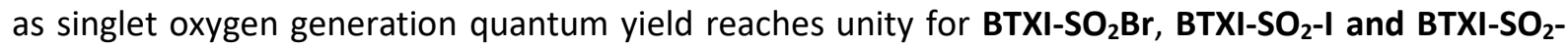
$\mathrm{BrBr}$. In contrast, substitution with $-\mathrm{NO}_{2}$ leads to a less straightforward evolution: the resulting BTXI$\mathrm{SO}_{2}-\mathrm{NO}_{2}$ molecule displays a singlet oxygen generation efficiency $\left(\phi_{\Delta}=0.57\right)$ that lies perfectly in between that of its parent BTXI-NO $\mathbf{N O}_{2}$ molecule $\left(\phi_{\Delta}=0.42\right)$ and that of $\mathbf{B T X I - S O _ { 2 }}\left(\phi_{\Delta}=0.72\right)$ suggestive of a distinct and perhaps concurrent photophysical mechanism of SOC enhancement in both cases. Hence, a detailed theoretical study was undertaken to investigate the nature of these mechanisms.

\section{Theoretical investigation of the ISC}

From an electronic point of view, ISC processes, occurring between singlet and triplet states, depend on the interplay of two parameters. The latter are directly involved in the equation 3 , that describes the density of probability of an ISC transition, considered herein between $\mathrm{S}_{1}$ and $\mathrm{T}_{1}$ states (Fermi Golden rule for a first order transition). ${ }^{47,48}$

$$
\Gamma_{S 1-T 1}=\frac{2 \pi}{\hbar}\left|\left\langle T_{1}\left|\widehat{H}_{S O}\right| S_{1}\right\rangle\right|^{2} \delta\left(E_{S 1}-E_{T 1}\right)
$$

Hence, the first parameter of importance is the SOC between these two states, which was computed based on the Douglas-Kroll Hamiltonian using the Dalton code. The second parameter influencing the efficiency of the crossing is the energy proximity between the two states. The SOC computed between 
the $S_{1}$ state and the two first triplet states (namely $T_{1}$ and $T_{2}$ ) along with the energy difference between these states are given in Figure 5 and Figure 6.
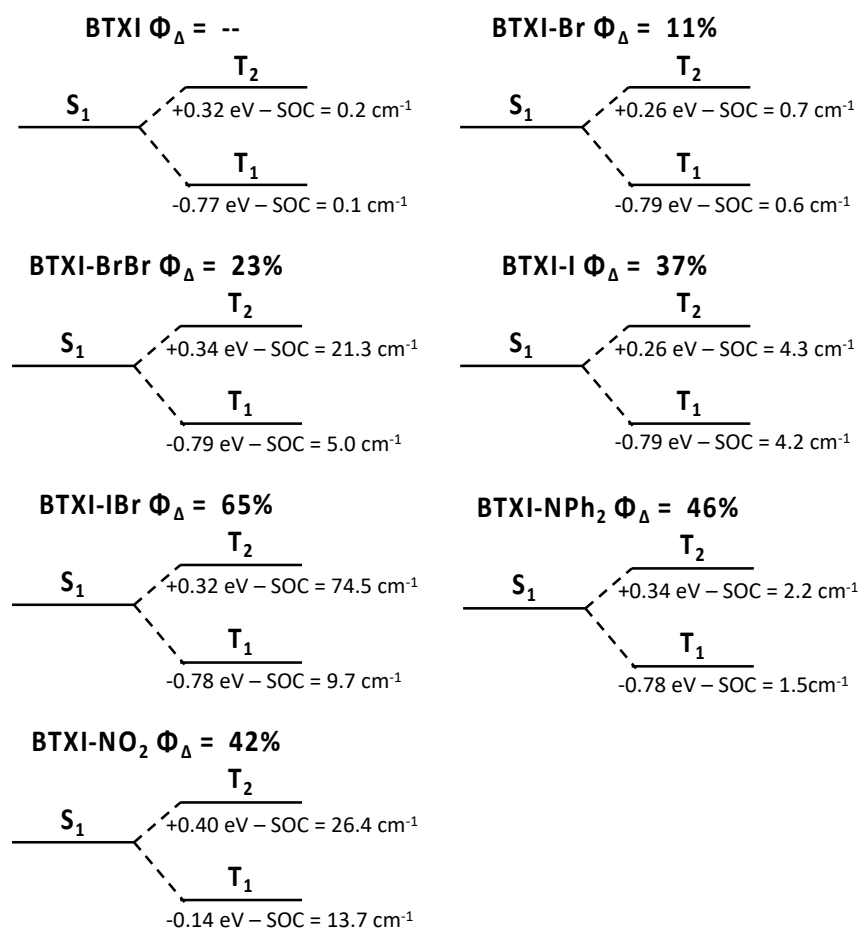

Figure 5. Computed spin-orbit coupling between the $\left(\mathrm{S}_{1}, \mathrm{~T}_{1}\right)$ and $\left(\mathrm{S}_{1}, \mathrm{~T}_{2}\right)$ states along with $\left(\mathrm{S}_{1}, \mathrm{~T}_{1}\right)$ and $\left(\mathrm{S}_{1}, \mathrm{~T}_{2}\right)$ energy difference of the BTXI series at the $\mathrm{S} 1$ stable geometry and at the B3LYP/PCM/aug-ccPVTZ level.
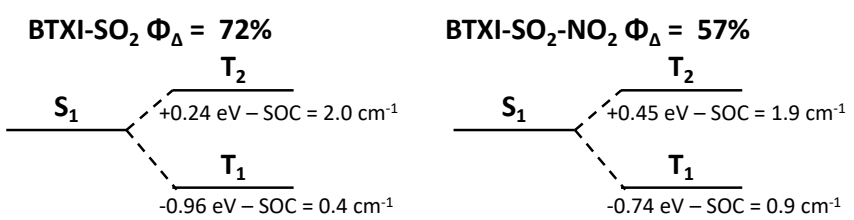

BTXI-SO $-\mathrm{Br} \Phi_{\Delta}=97 \%$

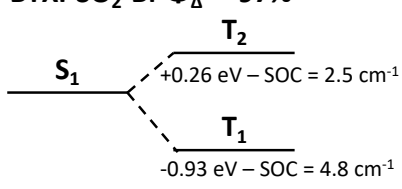

Figure 6. Computed spin-orbit coupling between the $\left(S_{1}, T_{1}\right)$ and $\left(S_{1}, T_{2}\right)$ states along with $\left(S_{1}, T_{1}\right)$ and $\left(\mathrm{S}_{1}, \mathrm{~T}_{2}\right)$ energy difference of the BTXI-SO $\mathrm{S}_{2}$ series at the $\mathrm{S}_{1}$ stable geometry and at the B3LYP/PCM/augcc-PVTZ level.

As a general observation, variations in singlet oxygen generation efficiency from one molecule to another are generally associated to a computed variation in SOC. This assumption holds true in the BTXI series, the large increase of efficiency of the dihalogenated compound as compared with their monohalogenated counterpart characterized experimentally being well corroborated by calculations. Besides the expected heavy atom effect that generally occurs upon halogen substitution, ${ }^{49}, 50$ which accounts here for the increase of SOC between BTXI-Br and BTXI-I on the one hand and between BTXI- 
$\mathrm{BrBr}$ and $\mathbf{B T X I - B r l}$ on the other, we can ascribe the extent of this phenomenon occurring in dihalogenated compounds to the distortion of the molecule induced by the halogen atom placed in the bay position. This distortion relates to a loss of planarity of the aromatic core, quantified by the $\Theta-$ angle in figure 7, which is much enhanced in dihalogenated compounds $\left(\Theta=23.1\right.$ and $29.3^{\circ}$ in $\mathbf{B T X I - B r B r}$ and $\mathbf{B T X I - B r I , ~ r e s p e c t i v e l y ) ~ c o m p a r e d ~ t o ~ t h e i r ~ m o n o h a l o g e n a t e d ~ a n a l o g u e s ~}\left(\Theta=2.5^{\circ}\right.$ in $\left.\mathbf{B T X I - B r}\right)$. To further and more specifically quantify the influence of distortion from planarity on the ISC, we also computed the SOC on molecules that were forced to be flat (CS point group imposed in the geometry relaxation) and compared to SOC computed for geometries relaxed without any constrains (Figure 7).

(a)

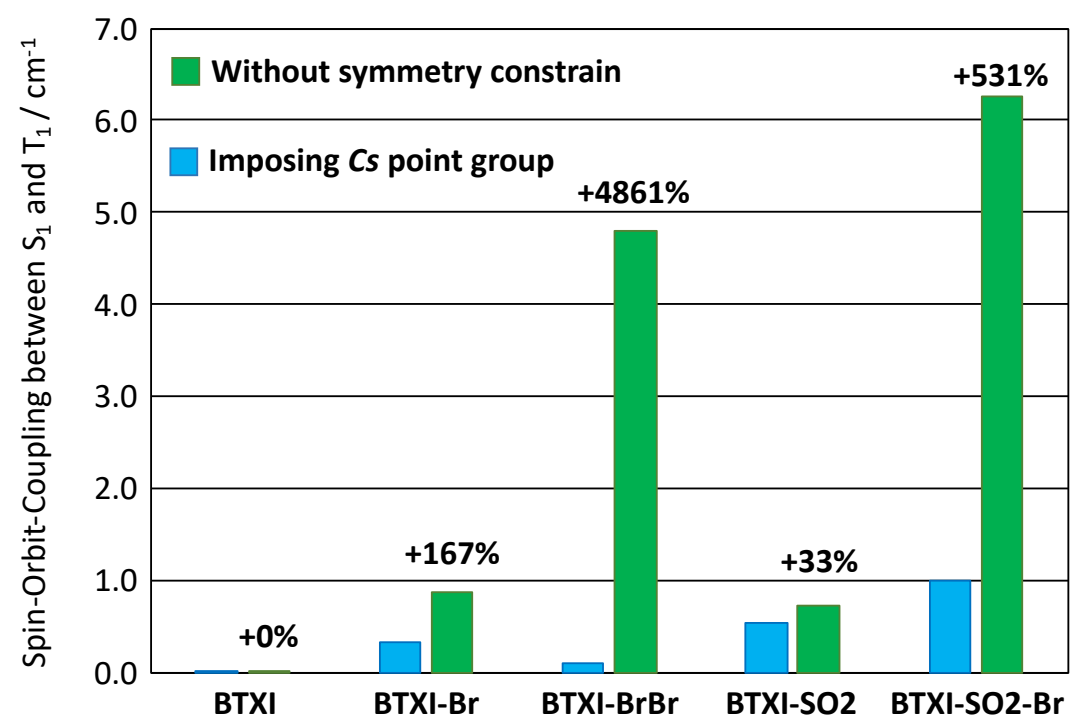

(b)

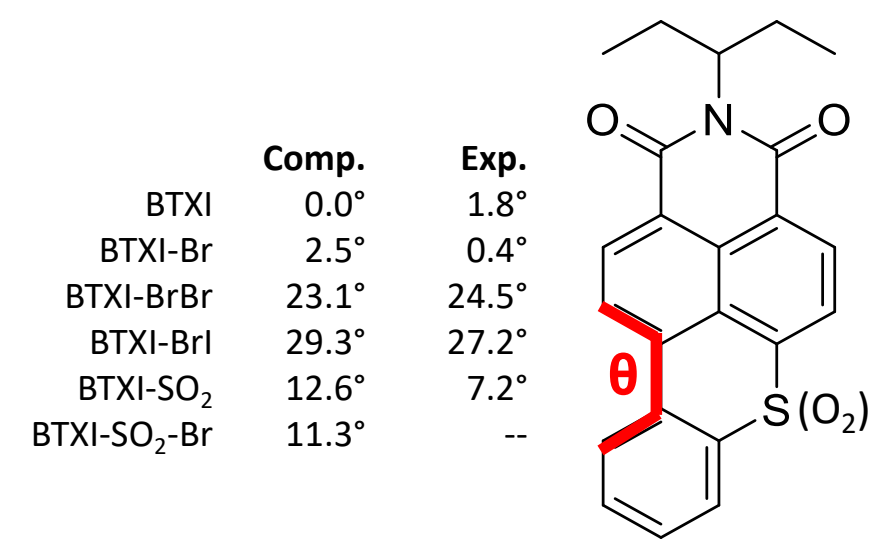

Figure 7. (a) Influence of the planarity loss of the aromatic system of the SOC between the $S_{1}$ and $T_{1}$ states. (b) Computed ground state and experimental (XRD) dihedral angle quantifying the loss of planearity of the molecule.

This investigation confirmed a strong influence of the geometry on the $\mathrm{SOC}$ for the dibrominated BTXI$\mathrm{BrBr}(+4861 \%)$ as compared to $\mathrm{BTXI-Br}(+167 \%)$. This influence of the distortion of the molecule on the SOC is sometimes called "spin-vibronic coupling". It has been discussed for instance by Monkmann et 
al. and Bredas et al. on molecules designed for TADF application and for distortions induced by the rotation between two parts of the molecule. ${ }^{51-53}$ To the best of our knowledge, this is the first time that such a large spin-vibronic coupling is reported for a distortion of an extended rigid $\pi$-system.

As discussed before in this article, the rotation of the $-\mathrm{NO}_{2}$ group at the $\mathrm{S} 1$ state induces a change in the nature of transition from $\pi->\pi^{*}$ to $n->\pi^{*}$. This is clearly visible by the large change in the shape of orbitals when computed at the $S_{0}$ and $S_{1}$ geometries (see Supporting Information). This excited state reorganisation induced symmetry breaking thus explains both the low fluorescence efficiency of this molecule (vide supra) and the large ISC as the symmetry forbidden $n->\pi^{*}$ transitions is known to strongly favor the SOC..$^{54,55}$

The strong ISC of the BTXI-SO ${ }_{2}$ series, highlighted by their large $\phi_{\Delta}$ experimentally measured, is also supported by TD-DFT with a strong SOC computed between the $S_{1}$ and $T_{1}$ states. For these molecules, the substituent-mediated aromatic distortion also slightly increases the SOC (+33\%), but contrary to the dihalogenated $\mathrm{BTXI}$ series (BTXI- $\mathrm{BrBr}$ and $\mathrm{BTXI-IBr}$ ), the forced $\mathrm{Cs}$ conformation of $\mathrm{BTXI}^{-\mathrm{SO}_{2}}$ already provides a strong SOC. In other words, the $-\mathrm{SO}_{2}$ group itself constitutes the main driving force for the ISC process. As mentioned before, a particularly intriguing experimental observation was the discrepancy between the additive effect observed on ISC efficiency when merging $-\mathrm{SO}_{2}$ and halogen substitution (BTXI-Br, $\phi_{\Delta}=0.11 ; \mathbf{B T X I} \mathrm{SO}_{2}, \phi_{\Delta}=0.72 ; \mathbf{B T X I}-\mathrm{SO}_{2}-\mathrm{Br}, \phi_{\Delta}=0.97$ ) and the corresponding

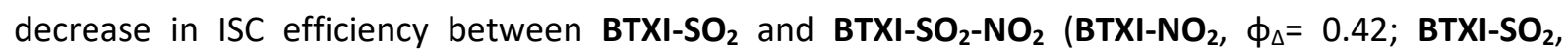
$\phi_{\Delta}=0.72 ; \mathrm{BTXI}-\mathrm{SO}_{2}-\mathrm{NO}_{2}, \phi_{\Delta}=0.57$ ). While the former is again well explained by distortion phenomena, the latter requires a less straightforward analysis. Interestingly, in the $\mathbf{B T X I - \mathbf { S O } _ { 2 }}$ series, the $\mathrm{T}_{2}$ state is

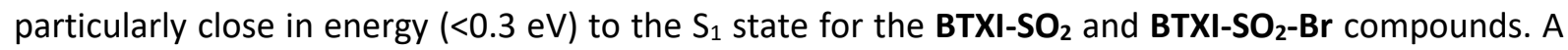
thermal population of $T_{2}$ from $S_{1}$ is thus possible opening a new path for triplet population and finally for singlet oxygen generation. For the $\mathbf{B T X I -} \mathbf{S O}_{2}-\mathbf{N O}_{2}$, the $\mathrm{T}_{2}$ state is further in energy $(>0.4 \mathrm{eV})$ and thus more difficult to populate by thermal energy that could explain the lower singlet oxygen generation efficiency of this last compound.

\section{Conclusions}

The study depicted herein confirms the exceptional potential of BTXI derivatives as triplet state harnesser and singlet oxygen photosensitizers. By playing on the nature and positions of the substituents on the $\pi$-conjugated structure, and simultaneously varying the nature and oxidation state of the heteroatoms involved in the heteroaromatic cycles, we were able to manipulate the ISC kinetics to afford in some cases a singlet oxygen generation quantum efficiency reaching unity. A systematic spectroscopic study coupled with computational modelling afforded a mean to study the effect of each individual modification of the skeleton on the global ISC process. We found out that halogenation of the molecule on the positions 5 and 11 rather than just one (5) resulted in much enhanced ISC kinetics, and that iodine were much more effective than bromine as substituents in that regard. The origin of the large increase of the ISC when both 5 and 11 positions are substituted stems from a spin-vibronic coupling itself activated by the distortion of the Pi-system due to the substituent. Even more interestingly, a strong improvement in the ISC could be achieved by simple oxidation of the thioxanthene moieties of the molecule, providing a very simple and efficient chemical mean to drive triplet formation in BTXI derivatives. Finally, combination of both strategies (sulfonation of the BTXI ring and halogenation of both positions) was highly efficient in maximizing ISC processes within the 
chromophore: as an illustration, a singlet oxygen generation efficiency $\phi_{\Delta}=1$ was monitored for two molecules of the series. We believe that this study constitutes a useful guideline for future works aiming at taking advantage of photoinduced triplet state generation in BTXI derivatives, which could find interesting applications in many topics ranging from molecular electronics to biomedicals.

1. H. Andreas and M. Klaus, Chem. Lett., 2006, 35, 978-985.

2. X. Zhan, A. Facchetti, S. Barlow, T. J. Marks, M. A. Ratner, M. R. Wasielewski and S. R. Marder, Adv. Mater., 2011, 23, 268-284.

3. J. Vollbrecht, H. Bock, C. Wiebeler, S. Schumacher and H. Kitzerow, Chem. Eur. J., 2014, 20, 12026-12031.

4. C. Ego, D. Marsitzky, S. Becker, J. Zhang, A. C. Grimsdale, K. Müllen, J. D. Mackenzie, C. Silva and R. H. Friend, J. Am. Chem. Soc., 2003, 125, 437-443.

5. C. P. Yu, R. Kimura, T. Kurosawa, E. Fukuzaki, T. Watanabe, H. Ishii, S. Kumagai, M. Yano, J. Takeya and T. Okamoto, Org. Lett., 2019, 21, 4448-4453.

6. X. Zhan, Z. a. Tan, B. Domercq, Z. An, X. Zhang, S. Barlow, Y. Li, D. Zhu, B. Kippelen and S. R. Marder, J. Am. Chem. Soc., 2007, 129, 7246-7247.

7. J. Wang and X. Zhan, Trends Chem., 2019, 1, 869-881.

8. Y. Lin, J. Wang, S. Dai, Y. Li, D. Zhu and X. Zhan, Adv. Energy Mater., 2014, 4, 1400420.

9. J. Warnan, J. Gardner, L. Le Pleux, J. Petersson, Y. Pellegrin, E. Blart, L. Hammarström and F. Odobel, J. Phys. Chem. C, 2014, 118, 103-113.

10. S. M. McAfee, S. V. Dayneko, P. Josse, P. Blanchard, C. Cabanetos and G. C. Welch, Chem. Mater., 2017, 29, 1309-1314.

11. A. Margineanu, J. Hofkens, M. Cotlet, S. Habuchi, A. Stefan, J. Qu, C. Kohl, K. Müllen, J. Vercammen, Y. Engelborghs, T. Gensch and F. C. De Schryver, J. Phys. Chem. B, 2004, 108, $12242-12251$.

12. M. Davies, C. Jung, P. Wallis, T. Schnitzler, C. Li, K. Müllen and C. Bräuchle, ChemPhysChem, 2011, 12, 1588-1595.

13. T. Weil, M. A. Abdalla, C. Jatzke, J. Hengstler and K. Müllen, Biomacromolecules, 2005, 6, 6879.

14. J. Qu, C. Kohl, M. Pottek and K. Müllen, Angew. Chem. Int. Ed., 2004, 43, 1528-1531.

15. T. Weil, T. Vosch, J. Hofkens, K. Peneva and K. Müllen, Angew. Chem. Int. Ed., 2010, 49, 90689093.

16. F. Würthner, Chem. Commun., 2004, 14, 1564-1579.

17. L. Chen, C. Li and K. Müllen, J. Mater. Chem., 2014, 2, 1938-1956.

18. J. Wu, D. He, L. Zhang, Y. Liu, X. Mo, J. Lin and H.-j. Zhang, Org. Lett., 2017, 19, 5438-5441.

19. P. H. Grayshan, A. M. Kadhim and A. T. Perters, J. Heterocycl. Chem., 1974, 11, 33-38.

20. A. M. Kadhim, K.-H. Mak and A. T. Peters, J. Soc. Dye. Colour., 1982, 98, 56-58.

21. P. Mao, X. Qian, H. Zhang and W. Yao, Dyes Pigm., 2004, 60, 9-16.

22. X. Qian, Y. Li, Y. Xu, Y. Liu and B. Qu, Bioorg. Med. Chem. Lett., 2004, 14, 2665-2668.

23. M. Danko, M. Mičušík, M. Omastová, J. Bujdák and D. Chorvát, Journal, 2013, 67, 18.

24. P. Josse, S. Li, S. Dayneko, D. Joly, A. Labrunie, S. Dabos-Seignon, M. Allain, B. Siegler, R. Demadrille, G. C. Welch, C. Risko, P. Blanchard and C. Cabanetos, J. Mater. Chem., 2018, 6, 761-766.

25. A.-J. Payne, N. A. Rice, S. M. McAfee, S. Li, P. Josse, C. Cabanetos, C. Risko, B. H. Lessard and G. C. Welch, ACS Appl. Energy Mater., 2018, 1, 4906-4916.

26. H. Yin, W. Zhu, Y. Xu, M. Dai, X. Qian, Y. Li and J. Liu, Eur. J. Med. Chem., 2011, 46, 3030-3037.

27. J. Nicolas, E. Khoshdel and D. M. Haddleton, Chem. Commun., 2007, 17, 1722-1724. 
28. J. M. Andrés Castán, L. Abad Galán, S. Li, C. Dalinot, P. Simón Marqués, M. Allain, C. Risko, C. Monnereau, O. Maury, P. Blanchard and C. Cabanetos, New J. Chem., 2020, 44, 900-905.

29. L. Zhang, Z. Huang, D. Dai, Y. Xiao, K. Lei, S. Tan, J. Cheng, Y. Xu, J. Liu and X. Qian, Org. Lett., 2016, 18, 5664-5667.

30. L. Zhang, K. Lei, J. Zhang, W. Song, Y. Zheng, S. Tan, Y. Gao, Y. Xu, J. Liu and X. Qian, MedChemComm, 2016, 7, 1171-1175.

31. M. Galland, T. Le Bahers, A. Banyasz, N. Lascoux, A. Duperray, A. Grichine, R. Tripier, Y. Guyot, M. Maynadier, C. Nguyen, M. Gary-Bobo, C. Andraud, C. Monnereau and O. Maury, Chem. Eur. J., 2019, 25, 9026-9034.

32. M. Galland, F. Riobé, J. Ouyang, N. Saleh, F. Pointillart, V. Dorcet, B. Le Guennic, O. Cador, J. Crassous, C. Andraud, C. Monnereau and O. Maury, European Journal of Inorganic Chemistry, 2019, 2019, 118-125.

33. C. Dalinot, P. Simón Marqués, J. M. Andrés Castán, P. Josse, M. Allain, L. Abad Galán, C. Monnereau, O. Maury, P. Blanchard and C. Cabanetos, Chemrxiv, 2020, DOI: 10.26434/chemrxiv.11865765.v1.

34. A. M. Brouwer, Pure Appl. Chem., 2011, 83, 2213-2228.

35. R. Schmidt, C. Tanielian, R. Dunsbach and C. Wolff, J. Photochem. Photobiol. A, 1994, 79, 1117.

36. M. J. Frisch, G. W. Trucks, H. B. Schlegel, G. E. Scuseria, M. A. Robb, J. R. Cheeseman, G. Scalmani, V. Barone, G. A. Petersson, H. Nakatsuji, X. Li, M. Caricato, A. V. Marenich, J. Bloino, B. G. Janesko, R. Gomperts, B. Mennucci, H. P. Hratchian, J. V. Ortiz, A. F. Izmaylov, J. L. Sonnenberg, Williams, F. Ding, F. Lipparini, F. Egidi, J. Goings, B. Peng, A. Petrone, T. Henderson, D. Ranasinghe, V. G. Zakrzewski, J. Gao, N. Rega, G. Zheng, W. Liang, M. Hada, M. Ehara, K. Toyota, R. Fukuda, J. Hasegawa, M. Ishida, T. Nakajima, Y. Honda, O. Kitao, H. Nakai, T. Vreven, K. Throssell, J. A. Montgomery Jr., J. E. Peralta, F. Ogliaro, M. J. Bearpark, J. J. Heyd, E. N. Brothers, K. N. Kudin, V. N. Staroverov, T. A. Keith, R. Kobayashi, J. Normand, K. Raghavachari, A. P. Rendell, J. C. Burant, S. S. Iyengar, J. Tomasi, M. Cossi, J. M. Millam, M. Klene, C. Adamo, R. Cammi, J. W. Ochterski, R. L. Martin, K. Morokuma, O. Farkas, J. B. Foresman and D. J. Fox, Gaussian 16 Rev. C.01, 2016, Wallingford, CT

37. C. Adamo and V. Barone, J. Chem. Phys., 1999, 110, 6158-6170.

38. D. Jacquemin, E. A. Perpète, I. Ciofini and C. Adamo, Acc. Chem. Res., 2009, 42, 326-334.

39. R. Krishnan, J. S. Binkley, R. Seeger and J. A. Pople, J. Chem. Phys., 1980, 72, 650-654.

40. J. Tomasi and M. Persico, Chem. Rev., 1994, 94, 2027-2094.

41. K. Aidas, C. Angeli, K. L. Bak, V. Bakken, R. Bast, L. Boman, O. Christiansen, R. Cimiraglia, S. Coriani, P. Dahle, E. K. Dalskov, U. Ekström, T. Enevoldsen, J. J. Eriksen, P. Ettenhuber, B. Fernández, L. Ferrighi, H. Fliegl, L. Frediani, K. Hald, A. Halkier, C. Hättig, H. Heiberg, T. Helgaker, A. C. Hennum, H. Hettema, E. Hjertenæs, S. Høst, I.-M. Høyvik, M. F. Iozzi, B. Jansík, H. J. A. Jensen, D. Jonsson, P. Jørgensen, J. Kauczor, S. Kirpekar, T. Kjærgaard, W. Klopper, S. Knecht, R. Kobayashi, H. Koch, J. Kongsted, A. Krapp, K. Kristensen, A. Ligabue, O. B. Lutnæs, J. I. Melo, K. V. Mikkelsen, R. H. Myhre, C. Neiss, C. B. Nielsen, P. Norman, J. Olsen, J. M. H. Olsen, A. Osted, M. J. Packer, F. Pawlowski, T. B. Pedersen, P. F. Provasi, S. Reine, Z. Rinkevicius, T. A. Ruden, K. Ruud, V. V. Rybkin, P. Sałek, C. C. M. Samson, A. S. de Merás, T. Saue, S. P. A. Sauer, B. Schimmelpfennig, K. Sneskov, A. H. Steindal, K. O. Sylvester-Hvid, P. R. Taylor, A. M. Teale, E. I. Tellgren, D. P. Tew, A. J. Thorvaldsen, L. Thøgersen, O. Vahtras, M. A. Watson, D. J. D. Wilson, M. Ziolkowski and H. Ågren, Wiley Interdiscip. Rev. Comput. Mol. Sci., 2014, 4, 269284.

42. W. A. d. Jong, R. J. Harrison and D. A. Dixon, J. Chem. Phys., 2001, 114, 48-53.

43. T. Nakajima and K. Hirao, Chem. Rev., 2012, 112, 385-402.

44. G. Bussi, D. Donadio and M. Parrinello, J. Chem. Phys., 2007, 126, 014101.

45. T. Le Bahers, C. Adamo and I. Ciofini, J. Chem. Theor. Comput., 2011, 7, 2498-2506.

46. C. Adamo, T. Le Bahers, M. Savarese, L. Wilbraham, G. García, R. Fukuda, M. Ehara, N. Rega and I. Ciofini, Coord. Chem. Rev., 2015, 304-305, 166-178. 
47. B. A. Heß, C. M. Marian, U. Wahlgren and O. Gropen, Chem. Phys. Letters, 1996, 251, 365-371.

48. X. Gao, S. Bai, D. Fazzi, T. Niehaus, M. Barbatti and W. Thiel, J. Chem. Theor. Comput., 2017, 13, 515-524.

49. P.-H. Lanoë, T. Gallavardin, A. Dupin, O. Maury, P. L. Baldeck, M. Lindgren, C. Monnereau and C. Andraud, Organic \& Biomolecular Chemistry, 2012, 10, 6275-6278.

50. B. Mettra, Y. Y. Liao, T. Gallavardin, C. Armagnat, D. Pitrat, P. Baldeck, T. Le Bahers, C. Monnereau and C. Andraud, Physical Chemistry Chemical Physics, 2018, 20, 3768-3783.

51. X.-K. Chen, S.-F. Zhang, J.-X. Fan and A.-M. Ren, J. Phys. Chem. C, 2015, 119, 9728-9733.

52. D.-H. Kim, A. D'Aléo, X.-K. Chen, A. D. S. Sandanayaka, D. Yao, L. Zhao, T. Komino, E. Zaborova, G. Canard, Y. Tsuchiya, E. Choi, J. W. Wu, F. Fages, J.-L. Brédas, J.-C. Ribierre and C. Adachi, Nat. Photonics, 2018, 12, 98-104.

53. G. Vampa, T. J. Hammond, M. Taucer, X. Ding, X. Ropagnol, T. Ozaki, S. Delprat, M. Chaker, N. Thiré, B. E. Schmidt, F. Légaré, D. D. Klug, A. Y. Naumov, D. M. Villeneuve, A. Staudte and P. B. Corkum, Nat. Photonics, 2018, 12, 465-468.

54. S. Rafiq, R. Yadav and P. Sen, J. Phys. Chem. A, 2011, 115, 8335-8343.

55. R. A. Vogt, C. Reichardt and C. E. Crespo-Hernández, J. Phys. Chem. A, 2013, 117, 6580-6588. 